mit der Beurteilung des Resultats nichts zu tun haben. Erstaunlich, dass derartige Rückzieher offenbar nicht nur auf die moderne schöne Literatur beschränkt sind!

INGRID SChELLbaCH

\title{
Religionsgeschichtliche Vorträge
}

Studies in Shamanism. Based on Papers Read at the Symposium on Shamanism Held at A bo on the $6^{\text {th }}-8^{\text {th }}$ of September, 1962. Ed. by Carl-Martin Edsman. Scripta Instituti Donneriani Aboensis I. Stockholm-Uppsala 1967. $186 \mathrm{~S}$.

Fatalistic Beliefs in Religion, Folklore and Literature. Papers Read at the Symposium on Fatalistic Beliefs Held at Abo on the $7^{\text {th }}-9^{\text {th }}$ of September, 1964. Ed. by Helmer Ringgren. Stockholm-Uppsala 1967. $186 \mathrm{~S}$.

In diesen boiden Bänden sind Vorträge veröffentlicht, die anlässlich von zwei Symposien (1962 und 1964) auf Initiative des Donner-Instituts in Turku ( $\AA$ bo) zu den im Titel genannten Themen gehalten wurden; eingangs wird darauf hingewiesen, dass Band I leider nicht alle Beiträge enthalte. Der Grund hierfür wird allerdings nicht bekanntgegeben.

In Band I macht Helmer Ringgren den Leser zunächst mit der Institution des Donner-Instituts bekannt, das 1957 durch eine im Testament von Uno Donner festgelegte Schenkung gegründet wurde. Nach dem Willen des Stifters, einem Anhänger und Förderer der Anthroposophie in Finnland, soll dieses Institut der wissenschaftlichen Erforschung der Religionsgeschichte und der Kulturgeschichte dienen. Das Institut besitzt eine fast vollständige Sammlung der Werke Rudolf Steiners und aller sonstigen anthroposophischen Autoren: diese sog. Steiner-Gedenkbibliothek zählt heute an 13.000 Bände. Ausserdem werden jährlich Stipendien zur Unterstützung von Forschern verteilt, die auf den genannten Gebieten arbeiten; die entsprechende Forschung wird auch anderweitjg unterstützt. Drittens wurde 1962 - zum ersten Mal an einer finnischen Universität - ein Lehrstuhl für vergleichende Religionsforschung an der schwedischen Universität von Turku, der Abo Akademi, eingerichtet. Viertens hat sich das Institut die Aufgabe gestellt, durch die Organisierung von Symposien zu Themen der Religionen Spezialisten zusammenzuführen und durch fruchtbaren Gedankenaustausch die einzelnen Sparten der Religionswissenschaften zu fördern. 
Die Artikel in Band I sind sämtlich in englischer Sprache geschrieben. Gustav Ränk bringt in seinem Beitrag »Der Schamanismus als Forschungsobjekt” einige methodische Gesichtspunkte. Er weist auf das quantitativ sehr umfangreiche Schrifttum bes. über die nordeurasische Version des Schamanismus hin, wo sich allerdings die unterschiedlichsten Definitionen und Interpretationen von Ursprung, Wesen und Aufgabe des Schamanismus finden. Verf. meint, man dürfe sich nicht nur auf die Untersuchung ron Herkunft und Entwicklung beschränken: Terminologie, Ausrüstung des Schamanen, seine Tätigkeit, verwandte religiöse Ideen und der funktionalistische Standpunkt, d.h. der Platz des Schamanen im sozial-ökonomischen und religiösen menschlichen Dasein, müssten mohr als bisher beachtet werden.

Die übrigen Aufsätzr bringen I)arstullungen oder Berichte von schamanistischen Vorstellungen bei verschiedenen Völkern, in verschiedenen Teilen der Welt: E. Holtved schreibt von den Eskimo, Åke Hultkrantz von einer nordamerikanischen schamanistischen Séance, H. Siiger von der 3. dänischen Expedition nach Zentralasien $1947-54$ zu den Kallash-Kafiren in Afghanistan; T. Schmid berichtet von schamanistischer Praktik in Nordnepal, Arvid $S$. Kapelrud entdeckt schamanistische Züge im Alten Testament, Jenő Fazekas äussert sich über den ungarischen Schamanismus. Carl-Martin Edsman nimmt die Gestalt der Catharina Fagerberg aus dem 18. Jh. zum Anlass, Verbindungspunkte zwischen volkstümlicher Heilkunde und schamanistischer Praktik zu suchen. Der Band schliesst mit dem Beitrag von Odd Nordland: Schamanismus als Erfahrung des "Unwirklichen».

Noch vielseitiger ist das Thema des zweiten Symposions: fatalistische Vorstellungen in Religion, Folklore und Literatur (letzteres wird zwar eher "rhetorisch" behandelt). Für den Begriff des Schicksals verfügen die sprachen ja über eine recht grosse Anzahl von Synonymen, die bei näherer Betrachtung alle ihre Berechtigung in kleinen Bedeutungsunterschieden haben und neben dem Schicksal als solchem auch das gute und das böse Geschick bezeichnen können (vgl. z.B. dt.: Schicksal, Geschick, Los, Vorsehung, Bestimmung, Fügung, Verhängnis, Fatum, Prädestination usw.).

Als Diskussionsgrundlage zitiert $H$. Ringgren im ersten Beitrag, "Das Problem des Fatalismus", vier grundlegende Interpretationen des Schicksals, wie Fr. Maass (Kiel) sie sieht: 1. Die "primitive" Interpretation, die dem Schicksal jeglichen Sinn abspricht; 2. Die heroische Ansicht, die im Schicksal ein unabwendbares Geset\% sieht, dem gegenüber der tragische Held standhält; 3. Die Leugnung, die Ablehnung des Schick- 
sals: die äusseren Umstände können nicht die Oberhand gewinnen und 4. die Akzeptierung des Schicksals, durchaus nicht nur charakteristisch für das Christentum. Aus dem Bereich des Schicksalsglaubens in Religionen werden die folgenden Themen behandelt: Göttliche und dämonische Notwendigkeit in der Orestie (C.-M. Edsman), Isis als Schicksalsgöttin (Jan Bergman), Islamischer Fatalismus (H. Ringgren). Der Aufsatz über vor- und nachchristlichen Schicksalsglauben in Skandinavien ( $\AA$ ke V. Ström) enthält ausser historischen Angaben die aufschlussreichen Eroebnisse zweicr kleinerer Umfragen (1963 und 1964) in Stockholm zum modernen Schicksalsglauben. Aus den im Text mitgeteilten Fragen und Antworten geht u.a. hervor. dass $56 \%$ an den Zufall glauben, $30 \%$ das Schicksal für das Geschehen verantwortlich michen, $35 \%$ glauben, dass komme, was da kommen soll, 26\% an Maskottchen glauben, $33 \%$ regelmässig und $58 \%$ manchmal Horoskope lesen; ausserdem ergaben die Umfragen. dass weibliche Personen mehr an das Schicksal, männliche mehr an den Zufall glauben.

İber den Schicksalsglauben in Südindien schreibt Carl Gustav Diehl, ïber entsprechende chinesische Vorstellungen äussert sich Gunnar Sjöholm. H. Siiger bringt auch hier einen Bericht von der 3. dänischen Expedition nach Zentralasien 1947/54: Das Schicksal in der Religion der Iuepcha, einem Ackerbauvolk im Himalaya im State Sikkim und in indischen Distrikten. Olof Petterson berichtet über dasselbe Thema in Ruanda-Urundi unter den Bantu in Afrika. Ivar Paulson schreibt über die Schicksalssecle bes. in den finnisch-ugrischen Volksreligionen. Mit der Erscheinungsform des Schicksals in der Literatur im weitesten Sinne setzt sich M. Brondsted kurz auseinander. K. Rob. V. Wikman bringt eine Zusammenfassung über Limnés Ideen hinsichtlich Vergeltung und Schicksal; es wird daranf hingewiesen, dass der Artikel vollständig als "Lachesis and Nemesis" veröffentlicht werden wird.

Matti Kuusi schreibt über die fatalistischen Züge in finnischen Sprichwörtern und kommt zu dem Schluss, dass die fatalistische Tradition in den jüngeren Sprichwortsammlungen eindentig zurückgeht und ersetzt wird durch den eigenen Willen, die persönliche Enerorie, im Sinne von »Jeder ist seines Glückes Schmied». Rolf Wilh. Brednich behandelt "Die osteuropäischen Volkssagen vom vorherbestimmten Schicksal». Seines Erachtens hat dieser überraschend einheitliche Komplex von Erzählgut seinen Platz weniger im Verzeichnis der Märchentypen als unter den Sagen "mit starkem Wirklichkeitsbezug und enger Bindung an den Volksglauben". Auch er spricht von einer Tendenz der Entdämonisierung der Erzählungen mit dem Schwinden der entsprechenden Glaubensvorstellungen. 
Den abschliessenden Beitrag liefert Gustav Henningsen mit einem kurzen Blick auf den systematischen Aspekt und den funktionalen Kontext des Fatalismus. Er macht den Vorschlag, man solle sich bei der Erforschung des Fatalismus auf eine Analyse der Situationen konzentrieren, in denen der Fatalismus zur Verwendung kommt. Dann würde sich nämlich zeigen, dass er in der Mehrheit der Fälle retrospektiv benutzt werde: wenn alle Mittel versucht sind, nichts mehr hilft, kommt man zur Resignation, zum Fatalismus. - Schicksalsglaube und Fatalismus sind jedoch Anfang und Endc einer ganz bestimmten Skala, zwischen denen eine Reihe von Vorstellungen Raum hat, die je nach dem Anteil der Wirksamkeit des persönlichen Willens, der eigenen Initiative einzugliedern sind.

Zu begrüssen ist m.E. ausser der Tatsache der Veröffentlichung dieser Vorträge auch der Verzicht auf eine Publikation der sich jeweils anschliessenden Diskussionen, die in ihrer Spontaneität nicht die notwendige Aussagekraft haben können.

INGRID SCHELLBACH

\section{Eine neue Einführung in die Turkologie}

Karl H. Menges, The Turkic Languages and Peoples. An Introduction to Turkic Studies. (Ural-Altaische Bibliothek, Band XV). Otto Harrassowitz, Wiesbaden 1968. XIV, 249 S.

Nach den durch die Zusammenarbeit mehrerer Forscher entstandenen Handbüchern der Turkologie, wie es die Philologiae Turcicae Fundamenta (Steiner, Wiesbaden $1959-$-) und das Handbuch der Orientalistik I 5: I (Brill, Leiden/Köln 1963) sind, bringt Harrassowitz hier eine Einleitung in die Turkologie, die eine Zusammenfassung der Ergebnisse der jahrzehntelangen Arbeit eines einzigen Forschers darstellt. Man kann wahrscheinlich feststellen, dass ein einziger Verfasser auch in seiner Subjektivität konsequenter ist als die Mitarbeiter eines Sammelwerkes, die oft in wesentlichen Fragen ganz inkompatible Standpunkte vertreten können. Prof. Menges hat sein Handbuch im Rahmen eines Projektes des American Council of Learned Societies i.J. 1962 abgefasst, vor der Drucklegung aber noch in gewissem Umfang ergänzt. In seinem Vorwort betont er, dass sein Buch keine vergleichende bzw. historische Grammatik der türkischen Sprachen sein will, sondern nur eine Einführung in die Turkologie. 